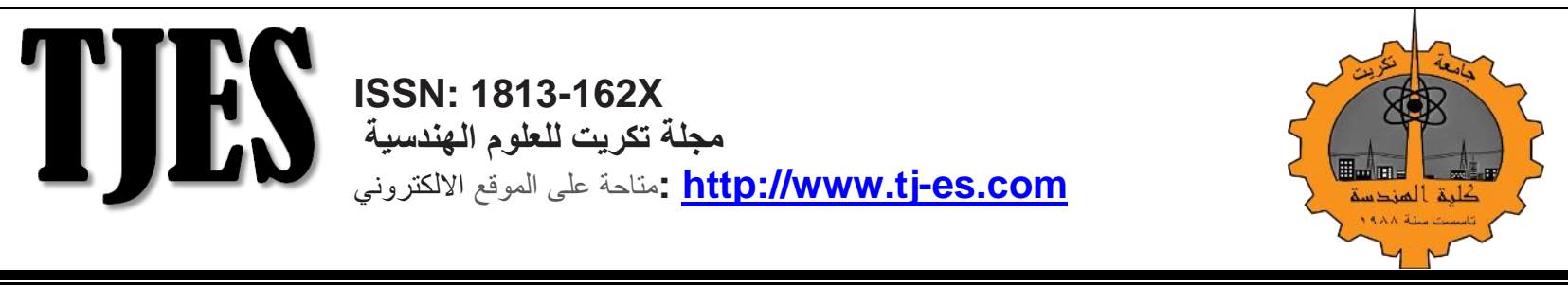

\title{
Production Hollow Ferrocement Beams Through Solid Waste Recycling
}

Aziz Ibrahim Abdulla

Salwa Hadi Ahmad

$$
\begin{aligned}
& \text { انتاج الاعتاب الفيروسمنتية المجوفة عن طريق تدوير النفايات الصلبة }
\end{aligned}
$$

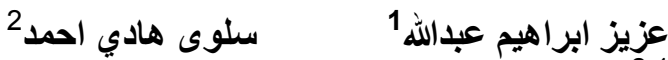

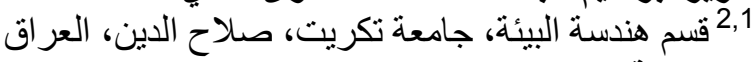

يتضمن البحث دراسة نظرية وعملية لسلوكية العتبات الفيروسمنتية المجوفة، والتي يتم عمل التجويف فيها

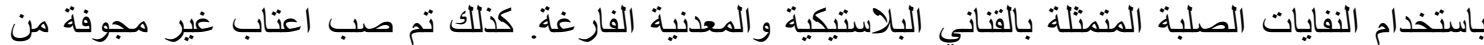

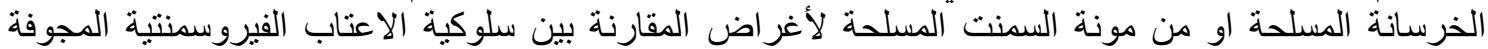

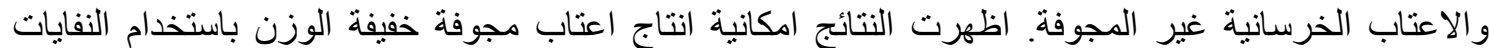
الصلبة وبكفاءة عالية. ان الاعتاب الفيروسمنتية المجوفة ذات مقاومة جيدة التيدة ومطيلية ومتانة عالية حيث النية الن استخدام

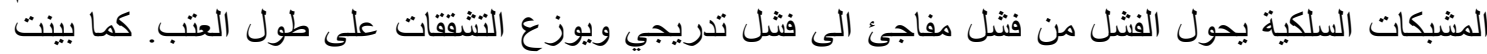

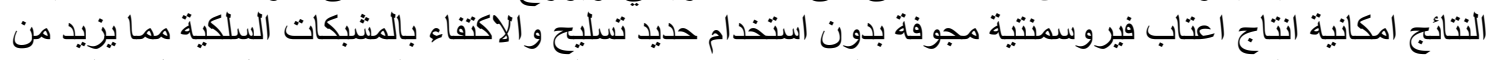

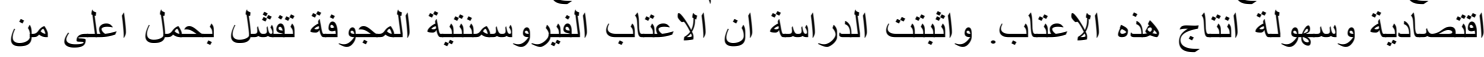

$$
\text { الكلمات الدالة: فيروسمنت، التصمي. نفايات صلبة، اعناب مجوفة، كاربون فايير. }
$$

\section{Abstract:}

Present research includes the study of theoretical and experimental behavior of hollow ferrocement beams, by using solid waste of empty plastic bottles and empty metal can. Solid reinforced concrete and reinforced cement mortar for the purposes of comparison between behavior of hollow ferrocement beams and solid beams were casted. The results showed the possibility and efficiently of producing lightweight hollow beams using solid waste. The hollow ferrocement beam have a good flexural strength resistance, toughness, and high ductility as use wired mesh converts a sudden failure to a gradual failure and distributes cracks along the beam. The results also show the possibility of the production of hollow ferrocement beams without rebar, where using of wire mesh only increasing the economic and easily produce these lightweight beams. The study proved that the ferrocement hollow beam fail under the load higher than the design load.

Keywords: Ferrocement, Hollow beam, Solid waste.

الفيروسمنت حيث درسوا نأثير كثافة واتجاه المشبكات

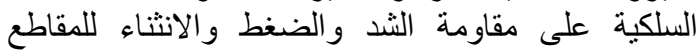

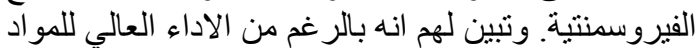

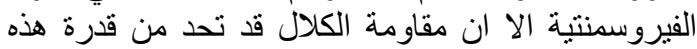

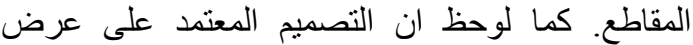

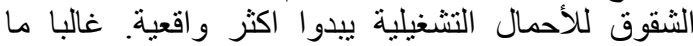

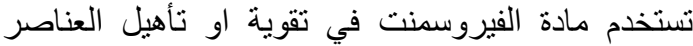

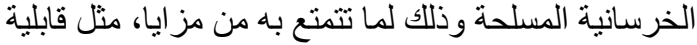

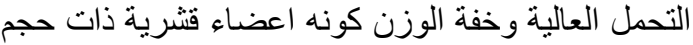

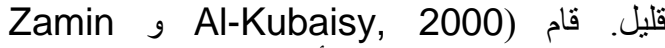

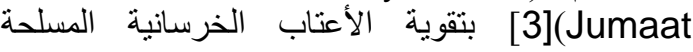

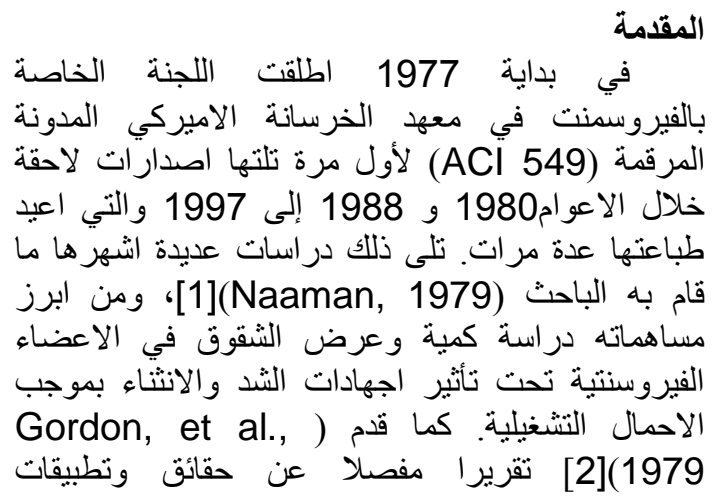


الغازية المعدنية او قناني العصائر البلاستيكية مما يعطي

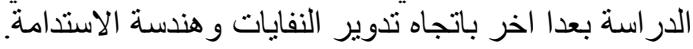

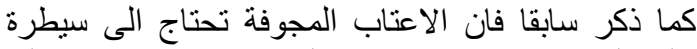

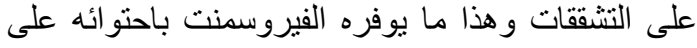

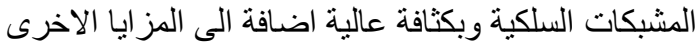

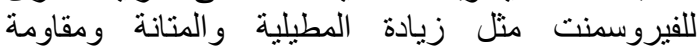
الصدمات و الديمومية العالية.

\section{اللهدف من الار اسة}

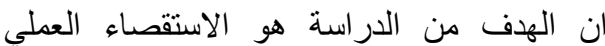
والنظري لسلوك الاعتاب الفيروسمنتية المجوفة الإنة، و المنتجةً باستخدام النفايات الصلبة. يتم در اسة سلوك الإي الاعتاب نظريا

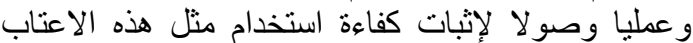

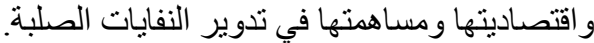

الدراسة النظرية

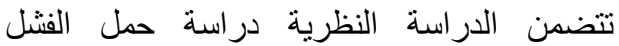

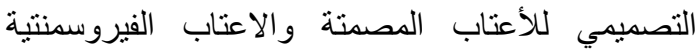
المجوفة. الشكل (1) يمثل مقطعا في الاعتاب الفيروسمنتية الفئية

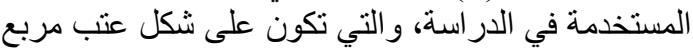

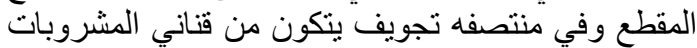

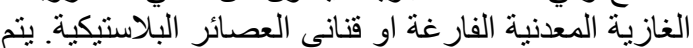

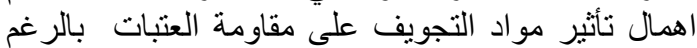

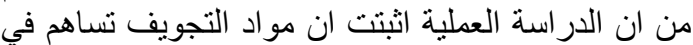
زيادة المقاومة. التجويف الوسطي يكون لتهن محاطا بالتسليح

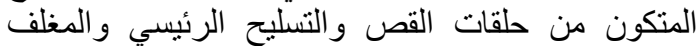
بطبقات من المشبكات السلكية بحيث بكون معدل الفئ سمك الفيروسمنت المحيط بالتجويف 5سم. يتم استخدام طريقة المئة المقاومة القصوى لحساب العزم الذي يحدث عنده الفثل

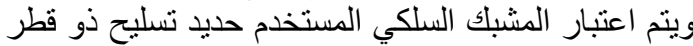

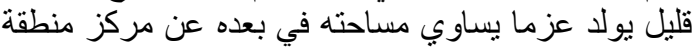

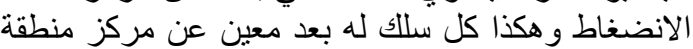

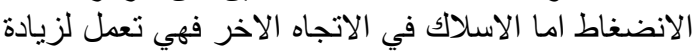

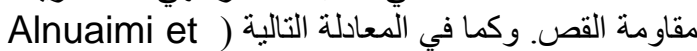
-:[5](al., 2008

$\mathrm{Pu}_{\text {the }}=4 \mathrm{Mn} /$

$\mathrm{Mn}=\operatorname{Asfy}(\mathrm{d}-\mathrm{a} / 2)$.

Or

$M n=\sum A s f y(d-a / 2)$

When using wire mesh حيث أن :

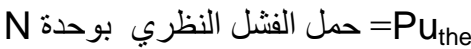
N.mm العزم الأقصى الذي يتحمله العتب بوحة بوحة =Mn

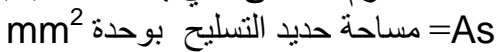

N/mm² إجهاد الخضوع لحديد التسليح بوحة لfy

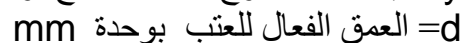
mm عمث منطقة الانضغاط الفعالة بوحدة لف =a =a

يتم مقارنة العزم الاقصى الذي تتحمله الاعتاب و المحسوب نظريا مع العزم الذي يتم الحصول عليه عمليا.
باستخدام المشبكات السلكية و لاحظا زيادة ملحوظة في قيم

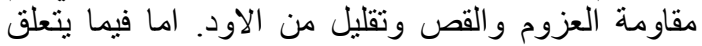

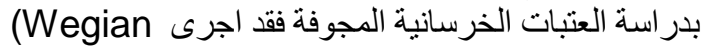
(4]and Almottiri, 2007) دراسة على الاعنى الاعتاب المجوفة التي تتتج باستخدام الكتل الخرسانية المجوفة

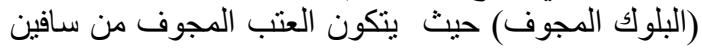

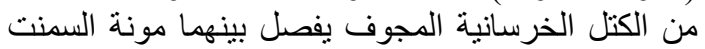

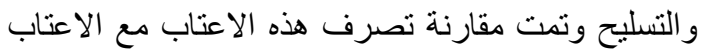

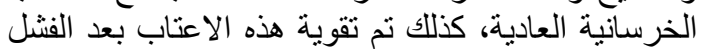

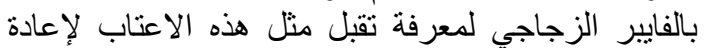

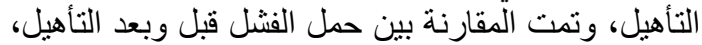

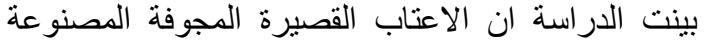

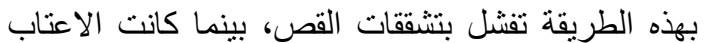

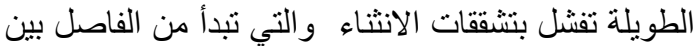

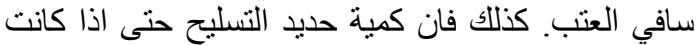
قليلة جدا فإنها تبقى مفيدة في زيادة قابلية التحمل. بعد اعلي اعادة

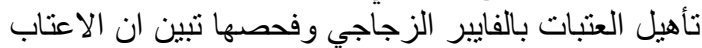
المجوفة تتقبل اعادة التأهيل افضل من الاعتاب الاعتيادية. أجرى ( Alnuaimi et al., 2008 (5) دراسة الأنة مقارنة لسلوكية كل من الاعتاب الخرسانية المصنة

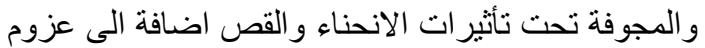

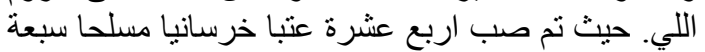

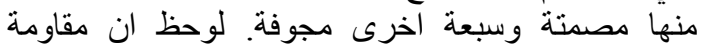

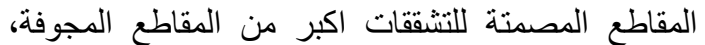

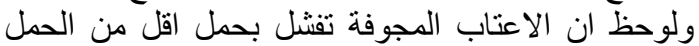

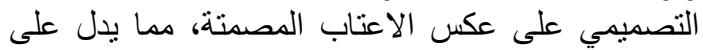
ضرورة السيطرة على التشققات في الاعتاب المجوفة.

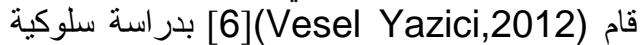

الاعمدة الخرسانية المسلحة والمجوفة، حيث قام بصد بـ 18

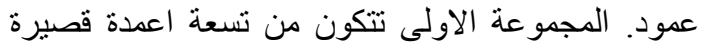

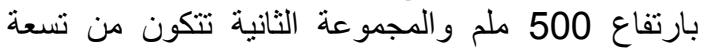

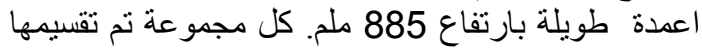

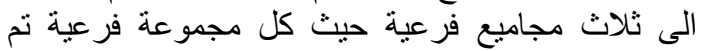

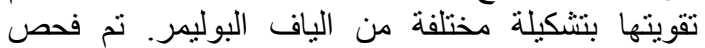

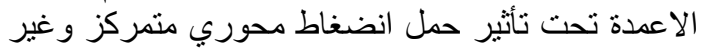
منمركز. تم مقارنة النتائج العملية بالحسابات النيات النظرية.

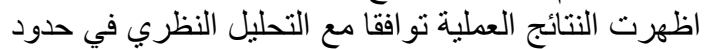

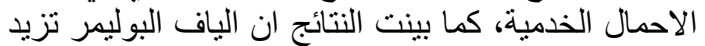
من كفاءة الاعمدة المجوفة بشئة بشكل كبير.

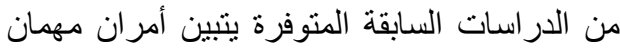
هما عدم وجود دراسات سابقة عن الاعتاب الفيروسمنتية المجوفة واهمية السيطرة على التشققات في الاعتاب الخرسانية المجوفة المبة

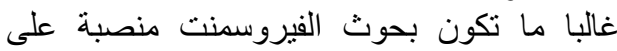

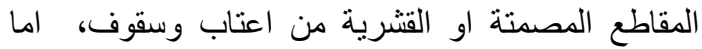
الاعتاب المجوفة فغالبا ما تكون من الخرية الخرسانة المسلحة.

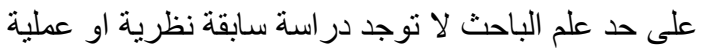

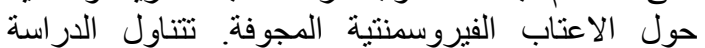
الحالية سلوكية الاعتاب الفيروسمنتية المجوفة الفية باستخدام

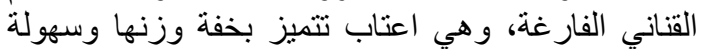

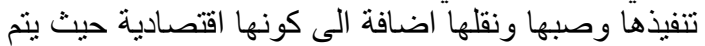

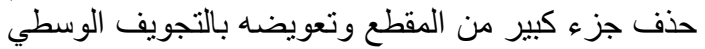
عن طريق استخدام النفايات الصلبة من قناني بني المشروبات 

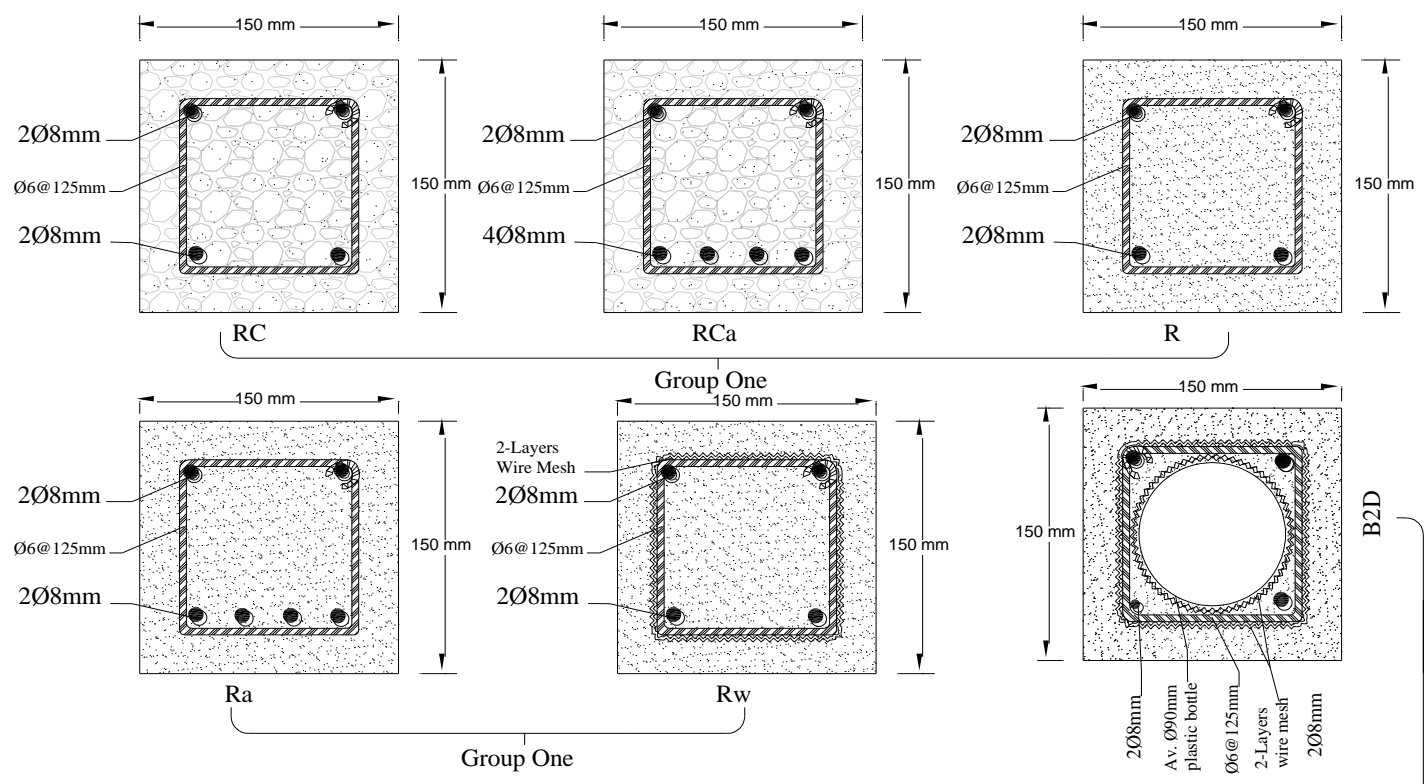

ลิ

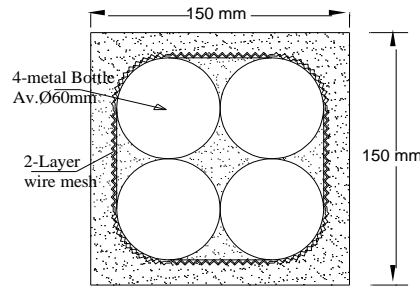

B3

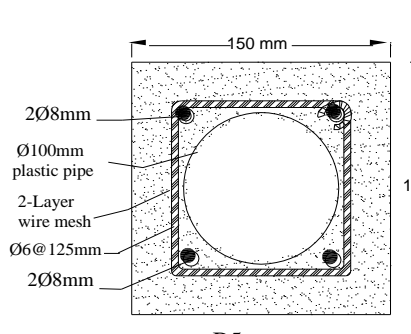

B5

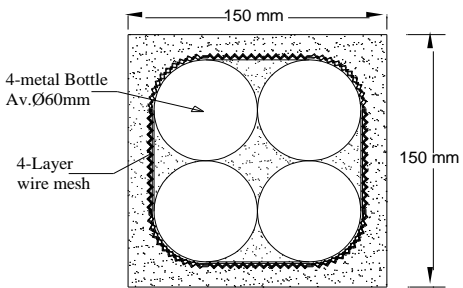

B4

Group Three

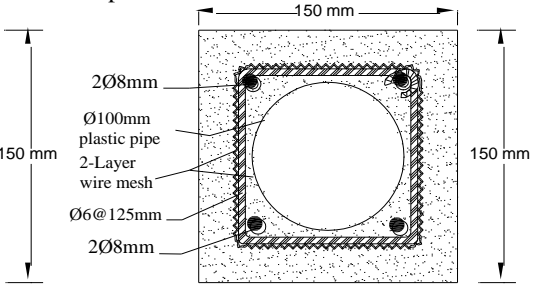

B6

Group Four

(1) تفاصيل مجاميع الأعتاب المستخدمة في البحث
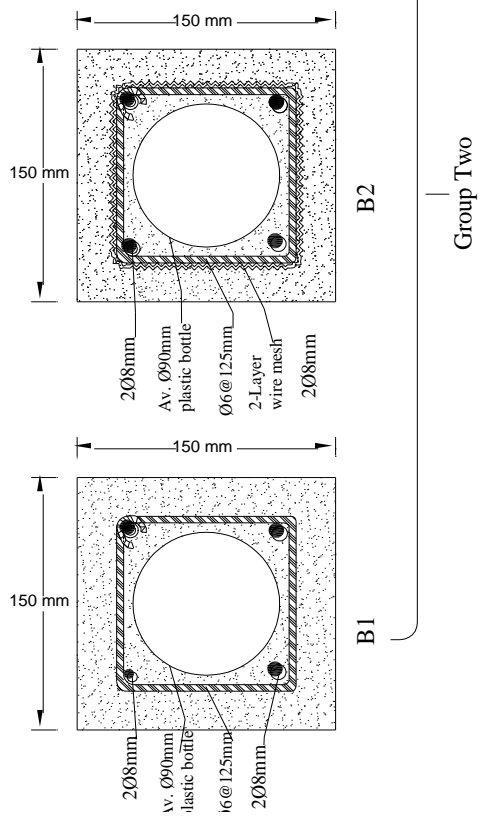

شكل (1) (1) (1) - (1)

خطة البحث العملية

ج- أعتاب من مونة السمنت يرمز لها R ومقدار تسليحها

$2 \varphi 8 \mathrm{~mm}$

دـ أعتاب من مونة السمنت يرمز لها Ra ومقدار تسليحها

.448mm

هـ- أعتاب من مونة السمنت يرمز لها Rw ومقدار تسليحها 248mm إضافة إلى طبقتين من المشبك

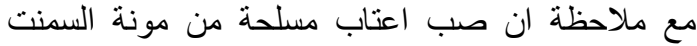

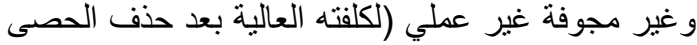

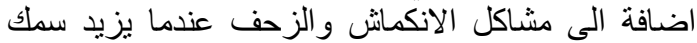

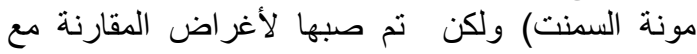

جميع الاعتاب التي تم صبها كانت ذات مقات مقطع

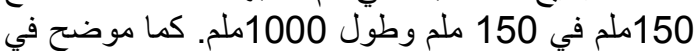

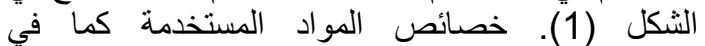
الملحق A. تتكون الاعتاب التي تم صبها من مجاميع

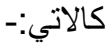

المجموعة الاولى:- تمثل المجموعة الاولى الاعتاب

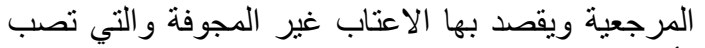

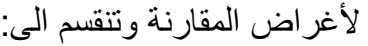

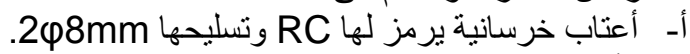

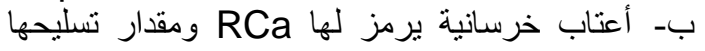

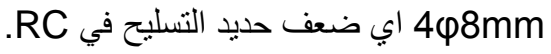




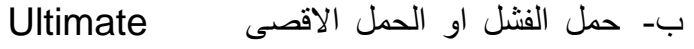
Load Pu

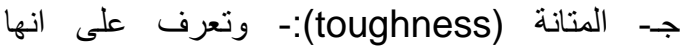

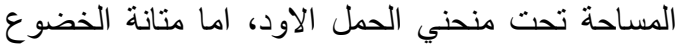

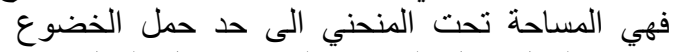

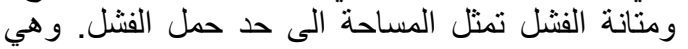

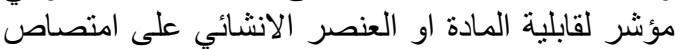

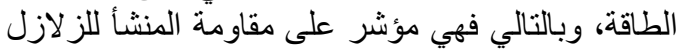

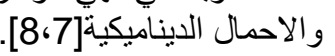

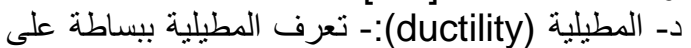

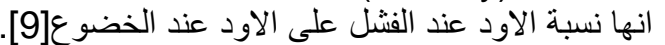
هـ- الصلابة (Stiffness):- تعرف الصاود الصلابة على انها ميل منحني الاود-الحمل وتحسب عندما نكون العلاقة خطية وعند حمل الخضوع او عند الخد 70\% من حمل

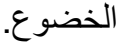

\section{النتائج والمناقشة}

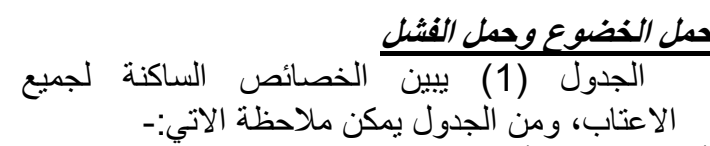

أ- بالنسبة للأعتاب المرجعية (غير المجوفة):- الاعتاب

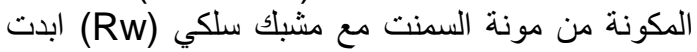

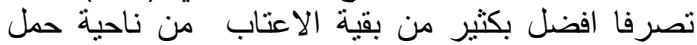

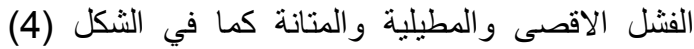
و الثكل (5) ويعود سبب ذللك الى ناثير المشبك الثيك السلكي

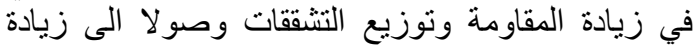
المطيلية. حيث كانت الفضل من الاعت الاعتاب المسلحة

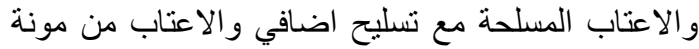
السنت مع تسليح اضافي ولكن بدون مشبك الكابك سلكي.

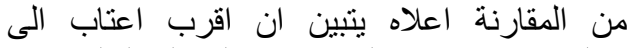

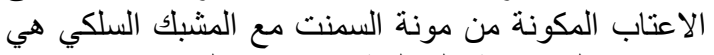

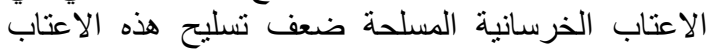

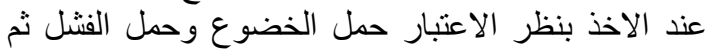

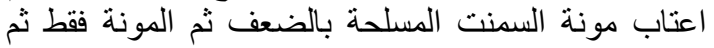

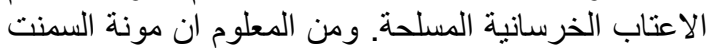

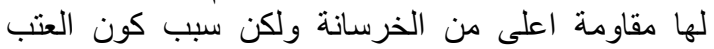
Rca الانكمانش و الزحف في حالة زيادة السمك وعدم وجود العرد المشبك السلكي.

ان الزيادة الكبيرة في حمل الخضوع وحمل الفشل الفيل

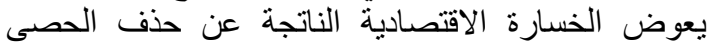

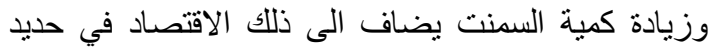

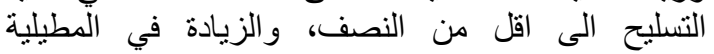

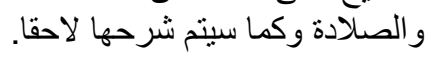

كما يلاحظ ان هناك اكتر اكثر من ما بمكن تسميته فثنل

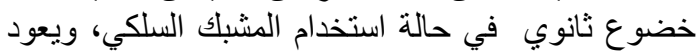

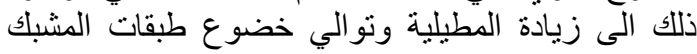
السلكي الواحدة تلو الاخرى. لمدئ

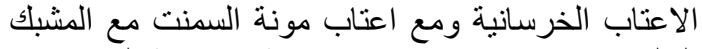

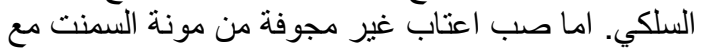
المشبك السلكي فهي عملية وقد اثتبت كفاءتها في زيادة المئدة المقاو مة و المتانة و المطيلية.

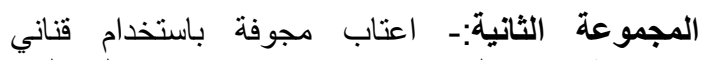
بلاسنيكية ويرمز لها B1 عندما تكون بدون مشبك سلكي

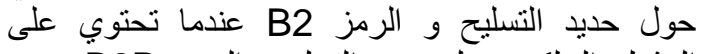

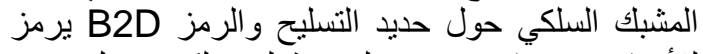
للأعتاب عندما تحتوي على مشبك سلكي حول حديد

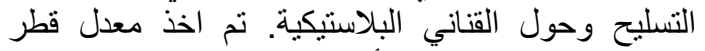

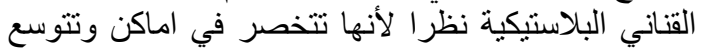

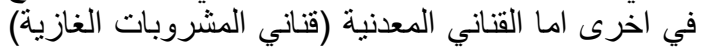
فان قطرها ثابت باستثناء تخصر الاني قليل في الاعلى

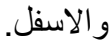

المجموعة الثالثة:- الثيء المميز لهذه المجموعة هو عدم

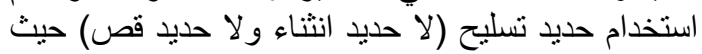

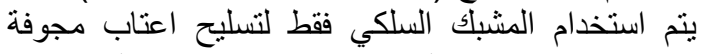

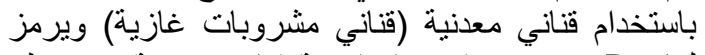

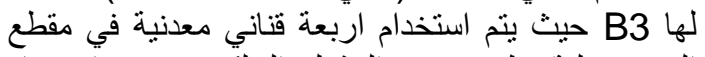
العتب مغلفة بطبقتين من المشبك السلكي وبدوني معند آستخدام حديد تسليح طولي او عرضي و الرمز B4 للأعناب التي مني تحتوي على اربع طبقات من آلمشبك السلكي. المجموعة الرابعة:- أعتاب مجوفة باستخدام انبوب

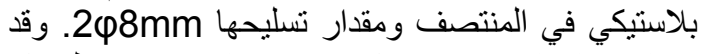

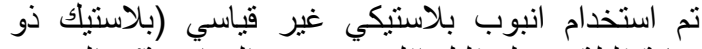

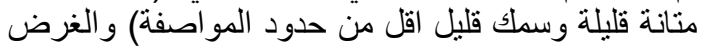

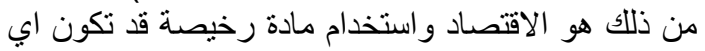

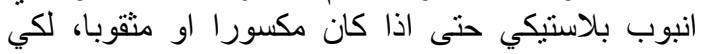
تدخل الفكرة ضمن تدوير النفايات وللمقارنة بلدي دين استخدام

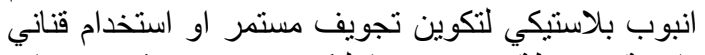

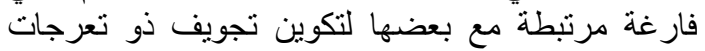

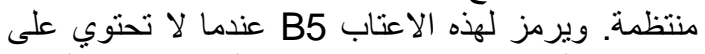

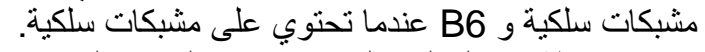

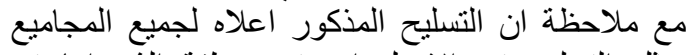

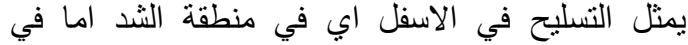

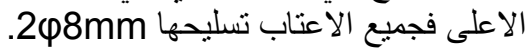

خطة الفحوصات

1- الخصائص الساكنة للأعتاب:- يتم فحص جميع الاعتاب بفضاء صافي قدره 80سم وبإسناد بسيط لخدي تحت حمل مركزي في المنتصف باستخدام جهاز الفحص العام Universal Test Machine (2) والذي يوضح طريقة الفحص وتوزيع التشققات في الثيكل

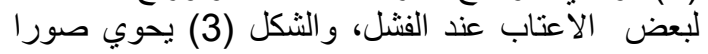

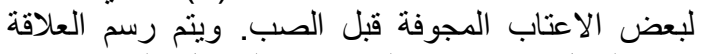

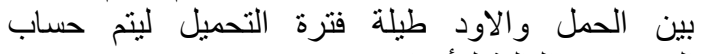
الخصائص التالية للأعتاب:-

أـ عمل الخضوع Yield Load Py 

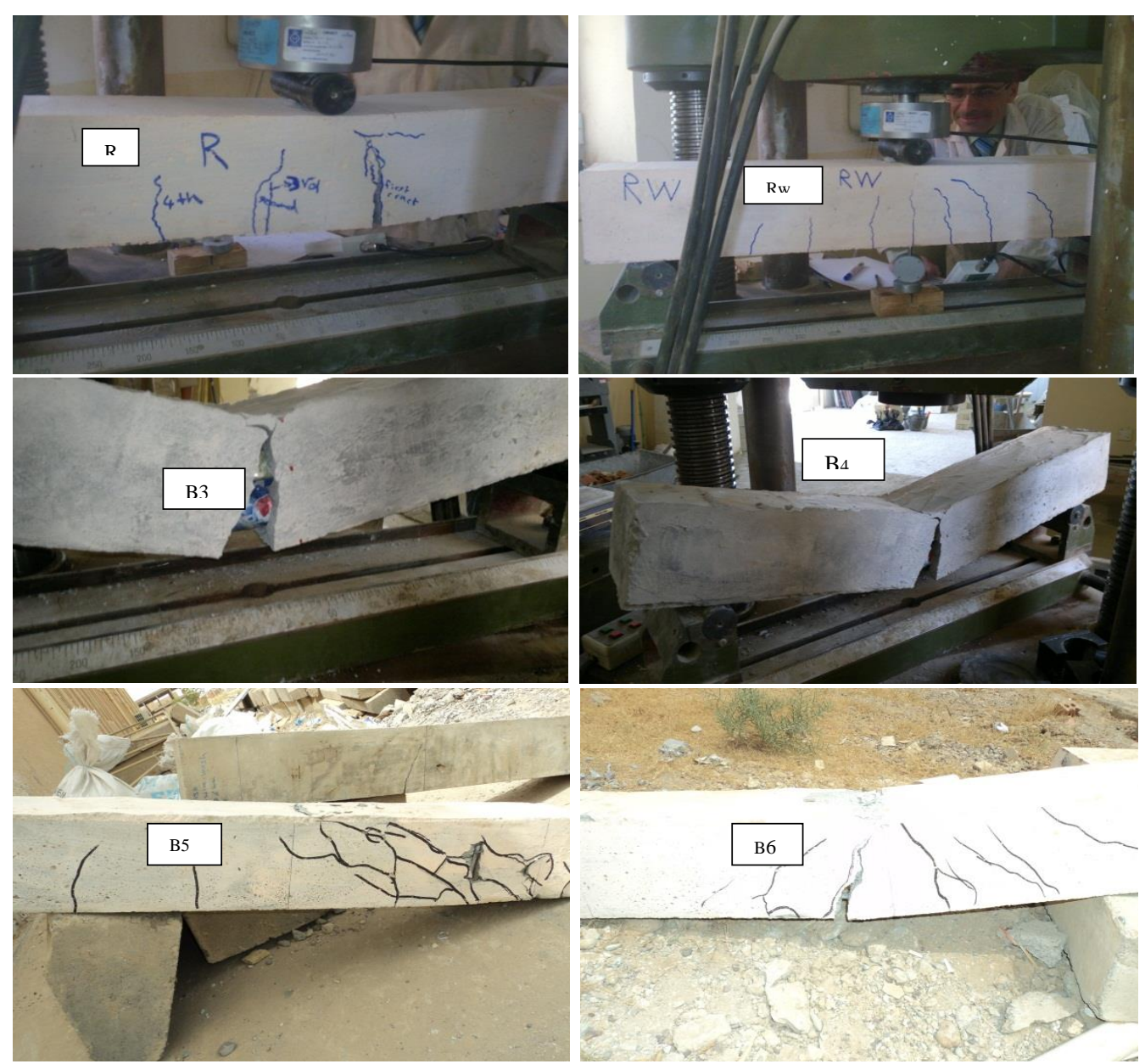

شكل (2) طريقة فحص الاعتاب تحت حمل مركزي ساكن مع شكل الفشل لبعضها

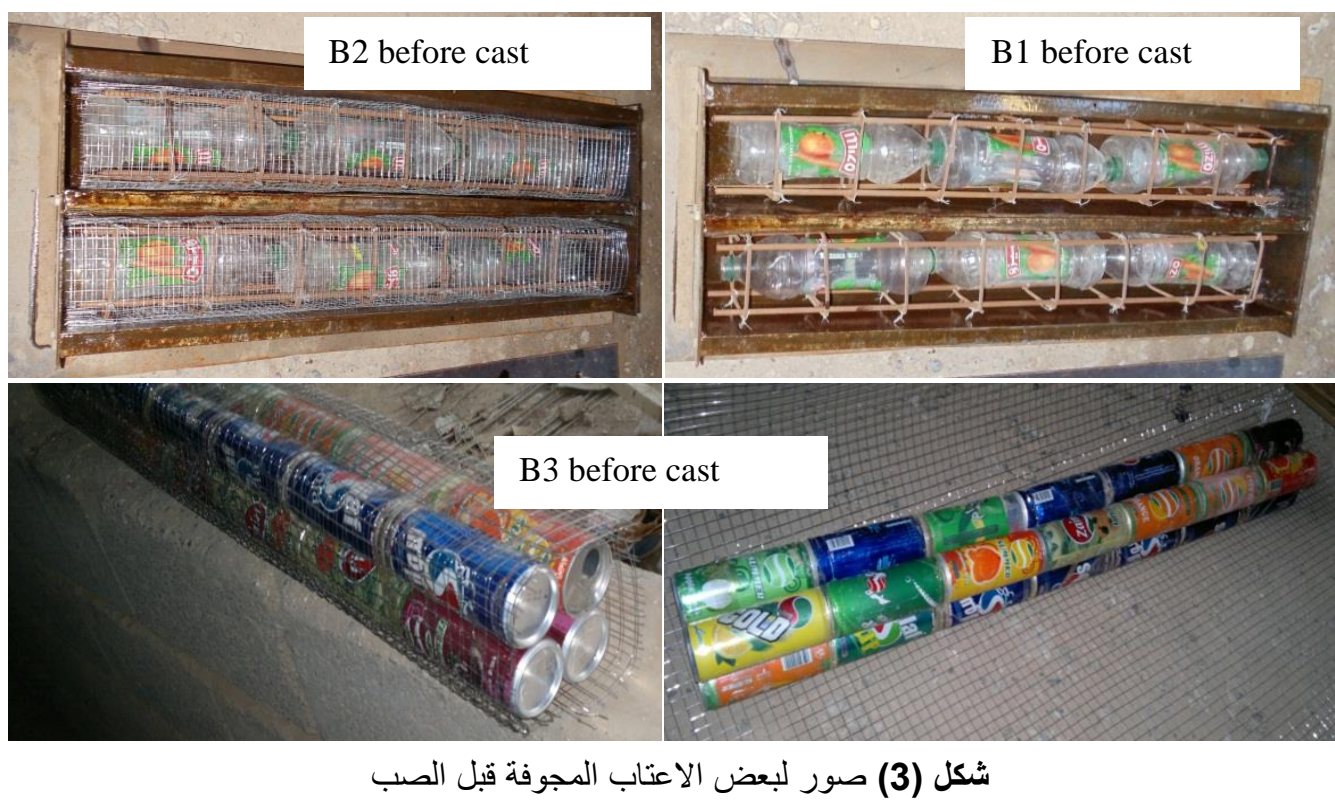


جدول (1) الخصائص السكونية للأعتاب

\begin{tabular}{|c|c|c|c|c|c|c|c|c|}
\hline \multirow{2}{*}{\multicolumn{2}{|c|}{ Beam Sym. }} & \multirow{2}{*}{ Type } & \multirow{2}{*}{$\begin{array}{l}\text { Py } \\
\mathrm{kN}\end{array}$} & \multirow{2}{*}{$\begin{array}{l}\mathrm{Pu} \\
\mathrm{kN}\end{array}$} & \multicolumn{2}{|c|}{ Toughness (N.m) } & \multirow[t]{2}{*}{ Ductility } & \multirow{2}{*}{$\begin{array}{c}\text { Stiffness } \\
\mathrm{N} / \mathrm{m}\end{array}$} \\
\hline & & & & & yield & Failure & & \\
\hline \multirow{5}{*}{$\begin{array}{c}\text { Group } \\
1\end{array}$} & $\mathrm{RC}$ & \multirow{2}{*}{$\begin{array}{l}\text { Ref. Concrete } \\
\text { beams }\end{array}$} & 4.1 & 22.5 & 0.24 & 27.553 & 16.167 & 33330 \\
\hline & $\mathrm{RCa}$ & & 9.45 & 49.7 & 1.181 & 138.67 & 16.4 & 37880 \\
\hline & $\mathrm{R}$ & \multirow{3}{*}{$\begin{array}{l}\text { Ref. Mortar } \\
\text { beams }\end{array}$} & 6.5 & 29 & 0.585 & 131.44 & 19.1 & \\
\hline & $\mathrm{Ra}$ & & 7.2 & 32.5 & 2.412 & & & \\
\hline & $\mathrm{Rw}$ & & 16.5 & 60 & 3.96 & 408.82 & 4 & 00 \\
\hline \multirow{3}{*}{$\begin{array}{c}\text { Group } \\
2\end{array}$} & $\mathrm{~B} 1$ & \multirow{3}{*}{ Plastic Bottle } & 8.5 & 25.7 & 1.125 & 38.43 & 9.2 & 32400 \\
\hline & B2 & & 9 & 32 & 1.215 & 52.84 & 9.63 & 33400 \\
\hline & B2D & & 14.1 & 35.75 & 2.89 & 92.44 & 9.93 & 34300 \\
\hline \multirow{2}{*}{$\begin{array}{c}\text { Group } \\
3\end{array}$} & B3 & \multirow{2}{*}{ Metallic can } & 4.0 & 5.9 & 0.48 & 47.1 & 52 & 16660 \\
\hline & B4 & & 4.78 & 7.1 & 0.55 & 74.1 & 74 & 20000 \\
\hline \multirow{2}{*}{$\underset{4}{\text { Group }}$} & B5 & \multirow{2}{*}{ Plastic Pipe } & 9 & 19.7 & 4.545 & 51.62 & 4.13 & 8910 \\
\hline & $\mathrm{B} 6$ & & 9.5 & 39.8 & 3.277 & 126.86 & 7.1 & 13760 \\
\hline
\end{tabular}

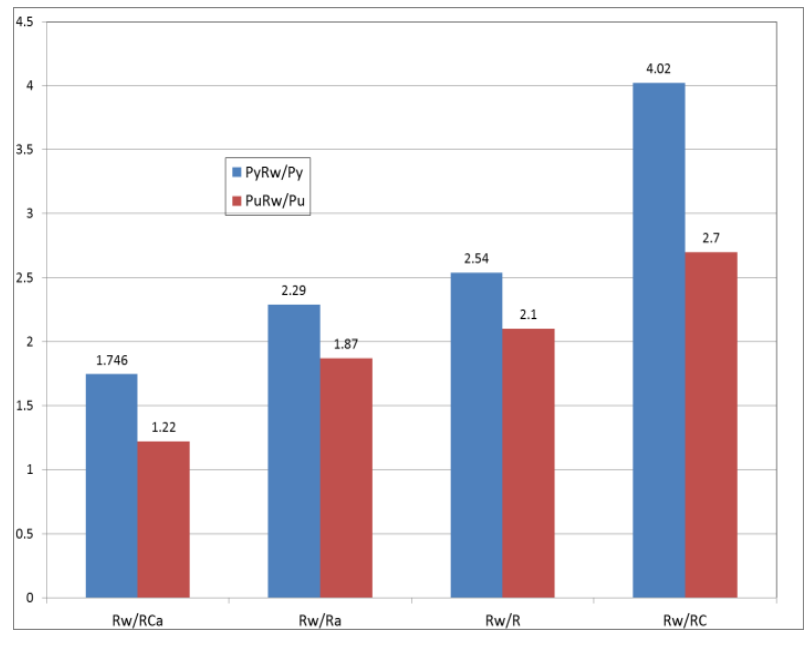

شكل (5) مقارنة اجهاد الخضوع و الفثل للأعتاب

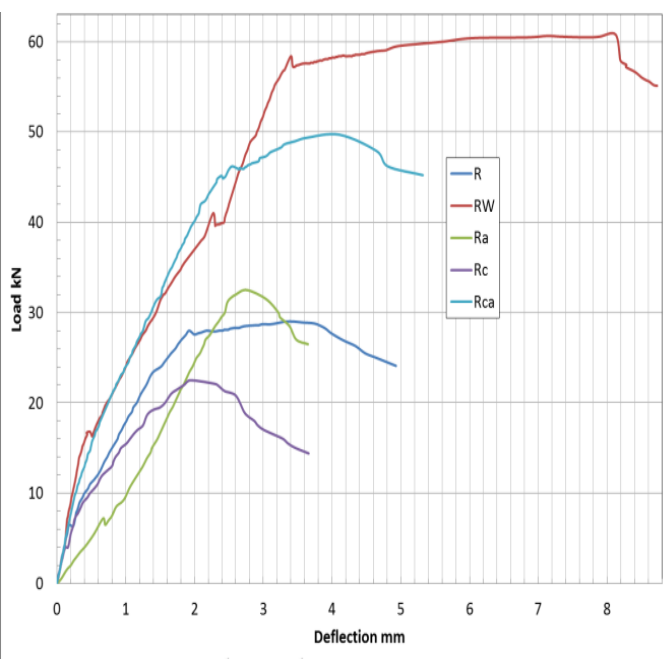

شكل (4) منحني الاود-الحمل للأعتاب
البلاستيكية يزيد من مقاومة اللي ومقاومة القص وهذا ما

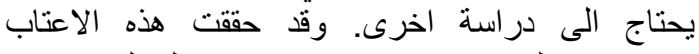
(B2D)

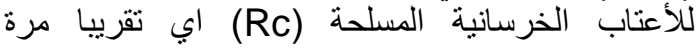

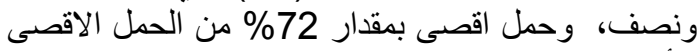

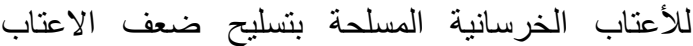

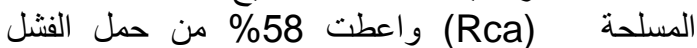
للأعتاب المتكونة من مونة السمنت مع مع مشبك من اعلكي (Rw)

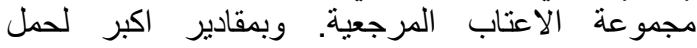
الخضوع مقارنة بجميع الاعتاب كما في الشكل (7).
ب- الأعتاب المجوفة باستخدام قناني بلاستيكية وباستخدام

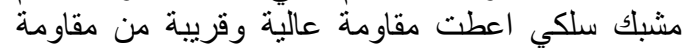
الاعتاب المسلحة غير المجوفة او اعتاب مونة المئة السمنت

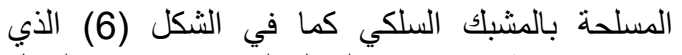
يوضح علاقة الاود مع الحمل لهذه الاعتاب ولئ والثكل

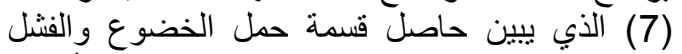
اللعتب B2D على ملى حمل الخضوع و الفشل للأعتاب

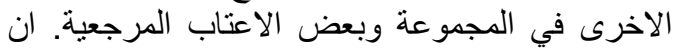

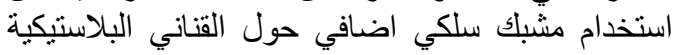

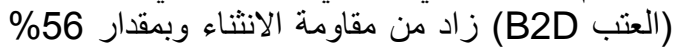

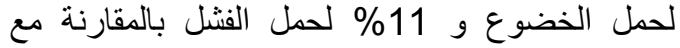

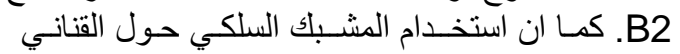



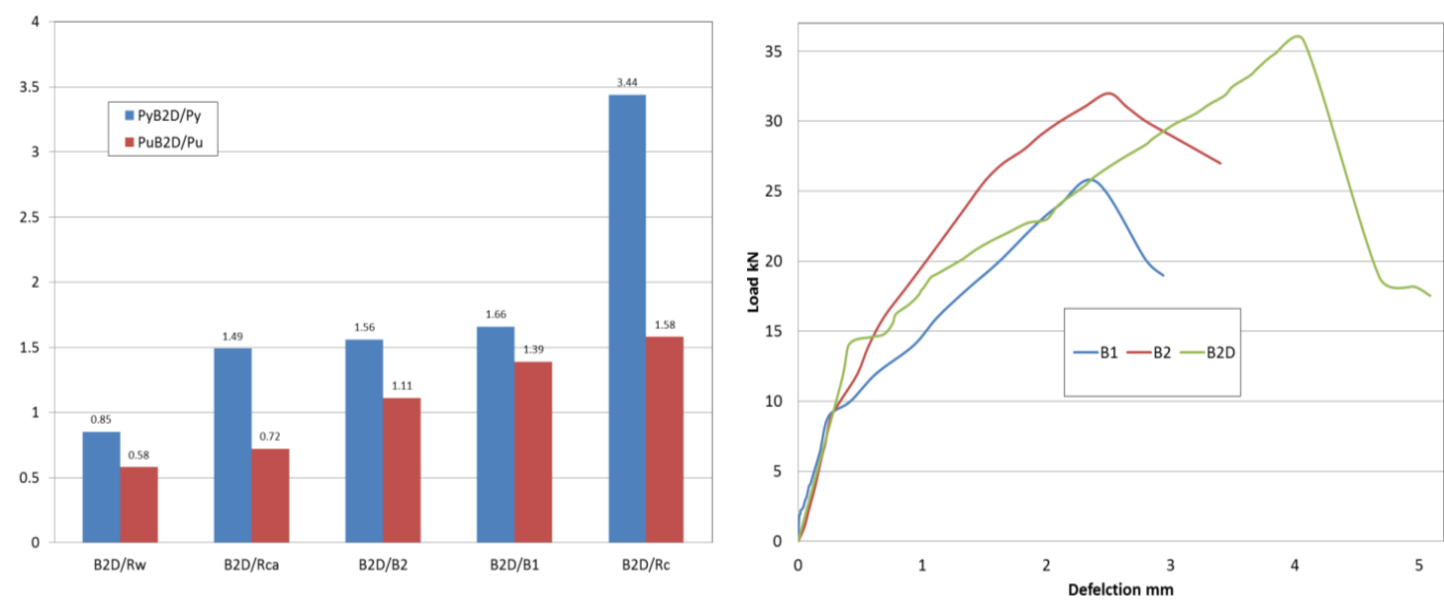

شكل (7) مقارنة اجهاد الخضوع و الفثل لاعتاب المجموعة الثانية ود-الحمل للاعتاب المرجعية

شكل (6) منحني الاود-الحمل لأعتاب المجموعة الثانية

بالنسبة للعتب B3. وهي نسب عالية خصوصا بالنسبة

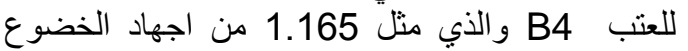

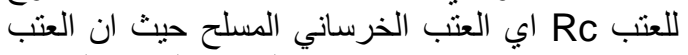

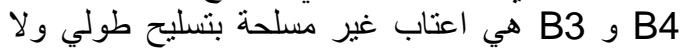
تسليح قص باستثناء المشبكات السلكية.

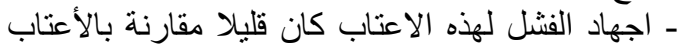
B4 حيث مثل اكبر قيمة للعتب بالان Rc و Rc و Rc

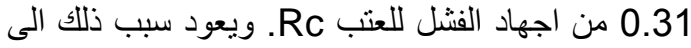
ان المشبكات السلكية تتقطع بقوة اقل بعد خضو علد عها بعكس

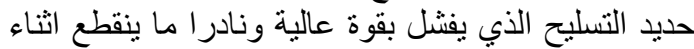

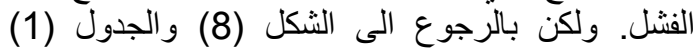

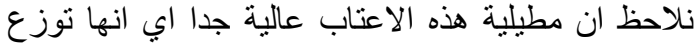

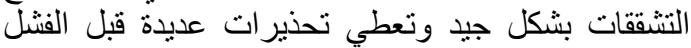
وبالتالي فهي مادة مطيلية Ductile. ويكية فيكن استخدام

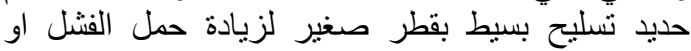

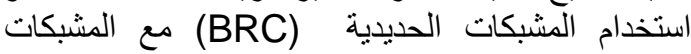
السلكية.

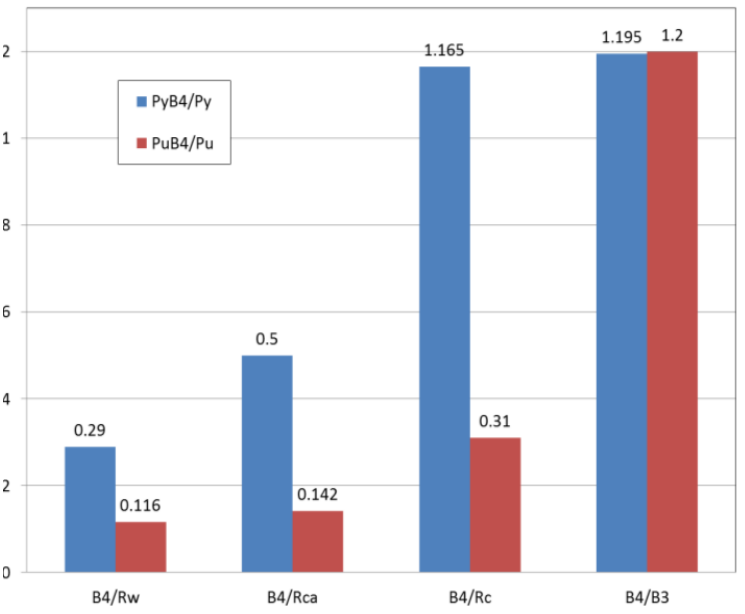

شكل (9) مقارنة حمل الخضوع و الفثل لاعتاب المجموعة الثالثة
ج- الأعتاب المنتجة بواسطة قناني معدنية رباعية في مقطع العتب تعطي مقاومة جيدة بحيث يمكن استخدام

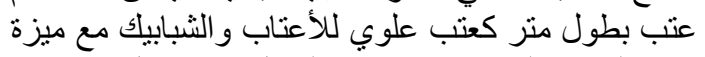

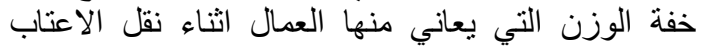

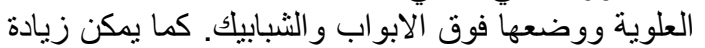

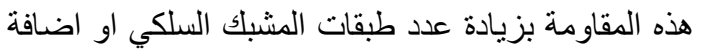

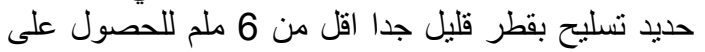
مقاومة عالية. و الثكل (8) يبين منحني الاودـ- الحمل لهذه

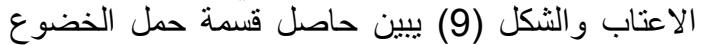

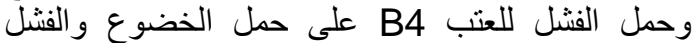

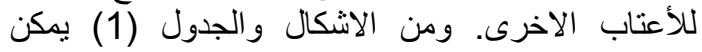
ملاحظة الاتي: - إجهاد الخضوع لهذه الاعتاب كان عاليا حيث مثل 0.29 و 0.5 و و Rca

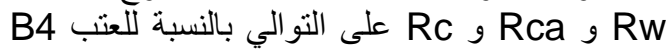
والنسب النالية 0.24 و 0.42 و و 0.97 و 0.97 من اجهاد الخضوع للأعتاب Rw و Rca و و Rc و R

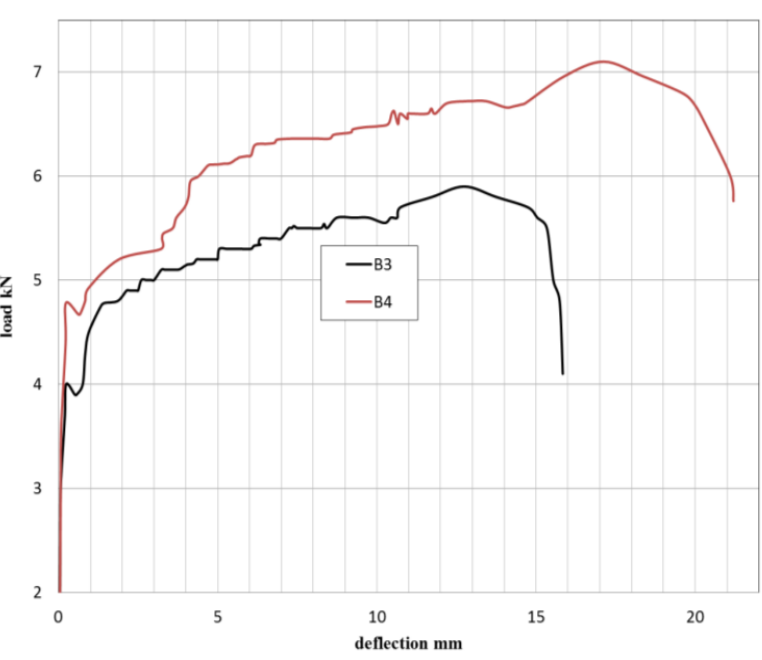

شكل (8) منحني الاود-الحمل لاعتاب المجمو عة الثالثة 
خضوع بمقدار 0.57 و 1 و 4.1 و 4.05 و 1.05 من حمل

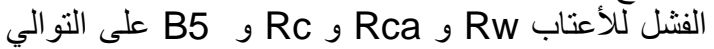
وحمل اقصى (حمل فثل) بمقدار 0.65 و 0.6 و 0.8 و 1.77

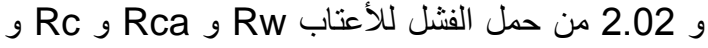
ع5 على التوالي. وهي نسب عالية ومقاربة ولكن اعلى

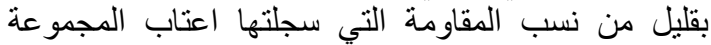

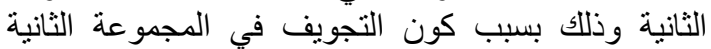

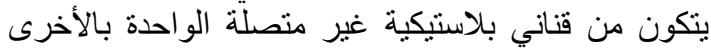

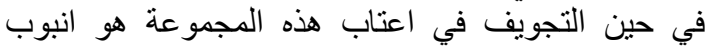
بلأستيكي مستمر واملس.

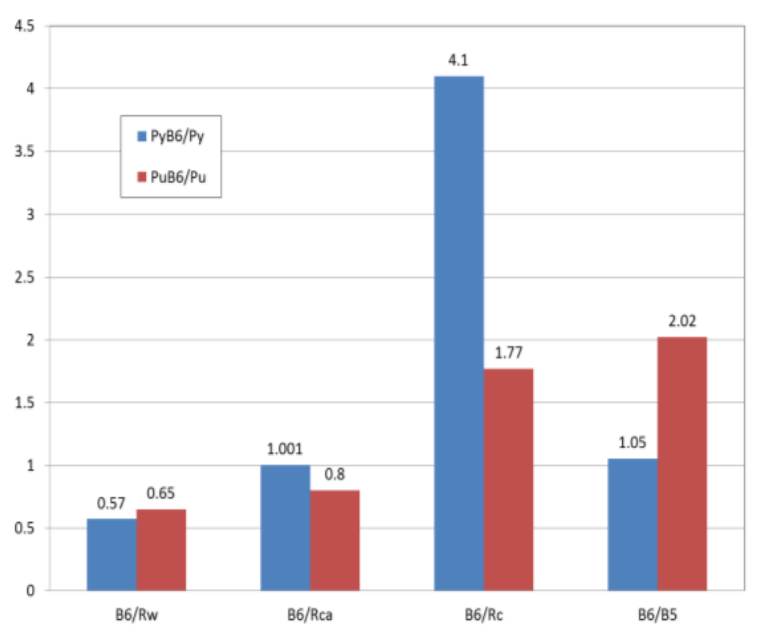

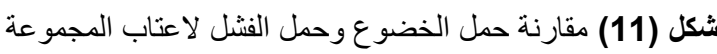

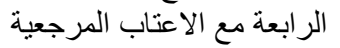

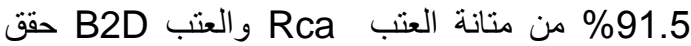

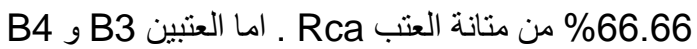

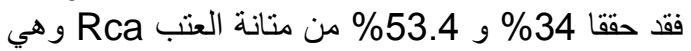

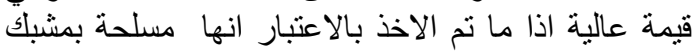

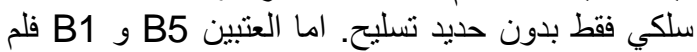
يعطيًا متانة فثل عالية لعدم تسليحهم بالمشبك السلكي.

\section{المطيلية Ductility}

يدل الثكل (12) بوضوح الى ان الاعتاب المجوفة

باستخدام قناني معدنية وبدون حديد تسليح رئيسي الاو حلقيات العات

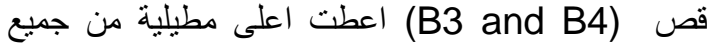
الاعتاب المجوفة وغير المجوفة، ويعود السبب في ذلكي اعلك الى الى

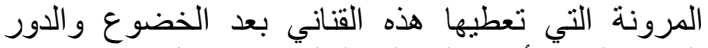
الكبير الذي يأخذه المشبك السلكي في ظل فئل غياب حديد

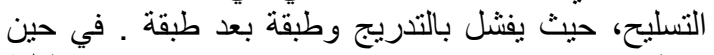

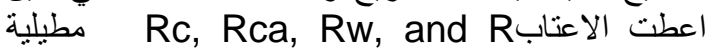

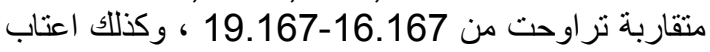

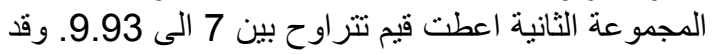

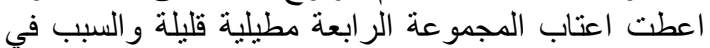

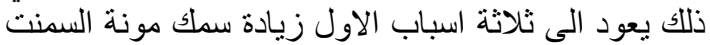

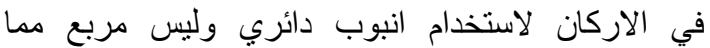

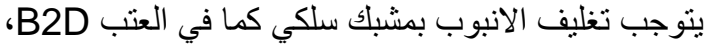
و الثاني عدم وجود ترابط بين الانبوب البلاستيكي ومونة
د- أعتاب المجموعة الرابعة :- وهي الاعتاب المجوفة بواب

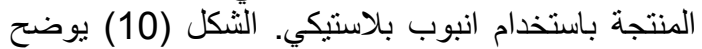

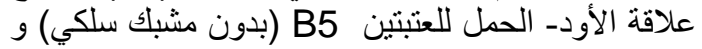

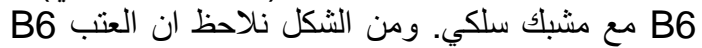

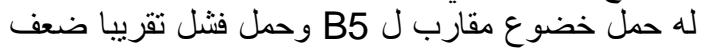

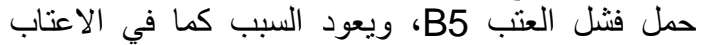
السابقة الى دور المشبك السلكي في توزيع التشققات الإنات وزيادة المقاومة و المطيلية. الما عند المقارنة مع الاعتاب المرجعية وبالرجوع (11) ولانية

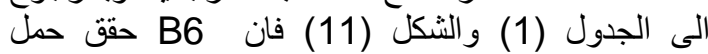

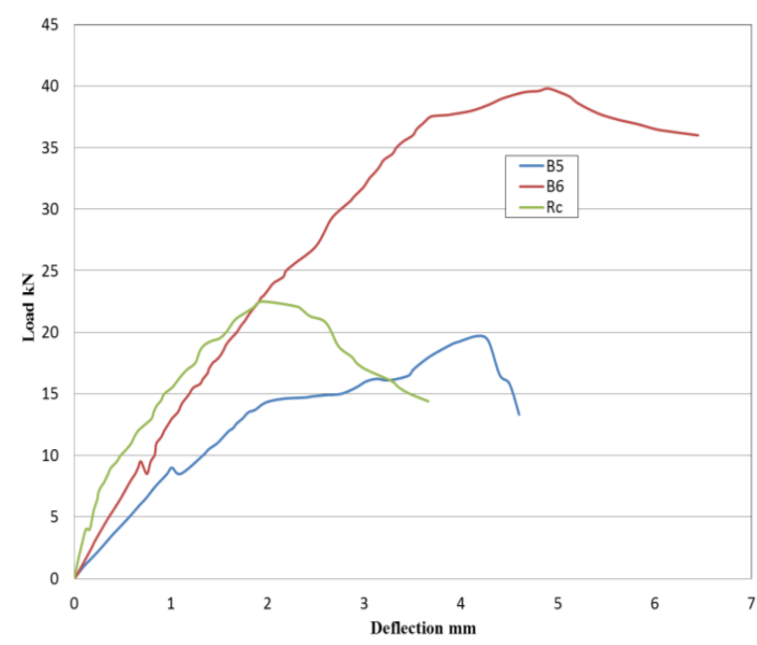

شكل (10) منحني الاود-الحمل لاعتاب المجموعة الر ابعة

(Toughness, المتانة والمطيلية والصلابة Ductility, and Stiffness)

الشكل (12) إضافة إلى الجدول (1) يبين منانة ومطيلية وصلابة الاعتاب.

- بالنسبة لمتانة الخضوع وصلابة والتي تمثل المساحة تحت منحني

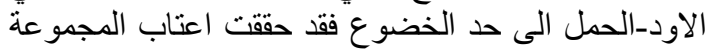

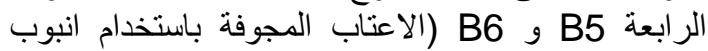
بلاستيكي) متانة خضوع عالية مقارنة الئة الأعتاب المرجعية

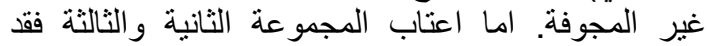

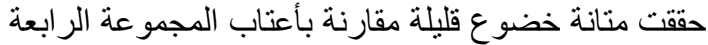
والاعتاب المرجعية المتكونة من مونة السمنت ولكتة ولكنها حققت متانة خضوع اعلى من الاعتاب الخرسانية المسلحة الأنة Rc

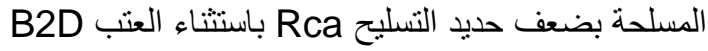
فقد حقق منانة خضوع تساوي تقريبا مرتين ونصف من بن

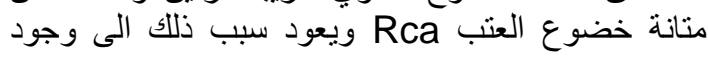
المشبك السلكي حول حديد التسليح وحول التبك القناني البلاستيكية. ـ أما بالنسبة لمتانة الفثل او المنانة القصوى (Ultimate)

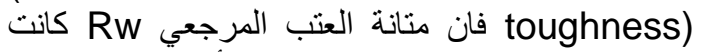

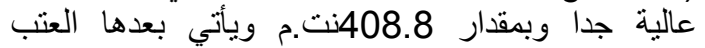
Rca الاعتاب المجوفة فقد حقق العتب B6 متانة قصوى بمقدار 
الصلابة Stiffness

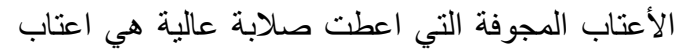

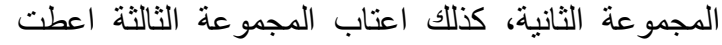

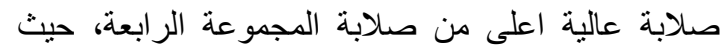

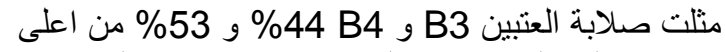

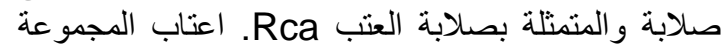
الرابعة لم تعطي صلابة عالية للأسباب المذكور في فقرة

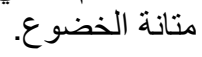

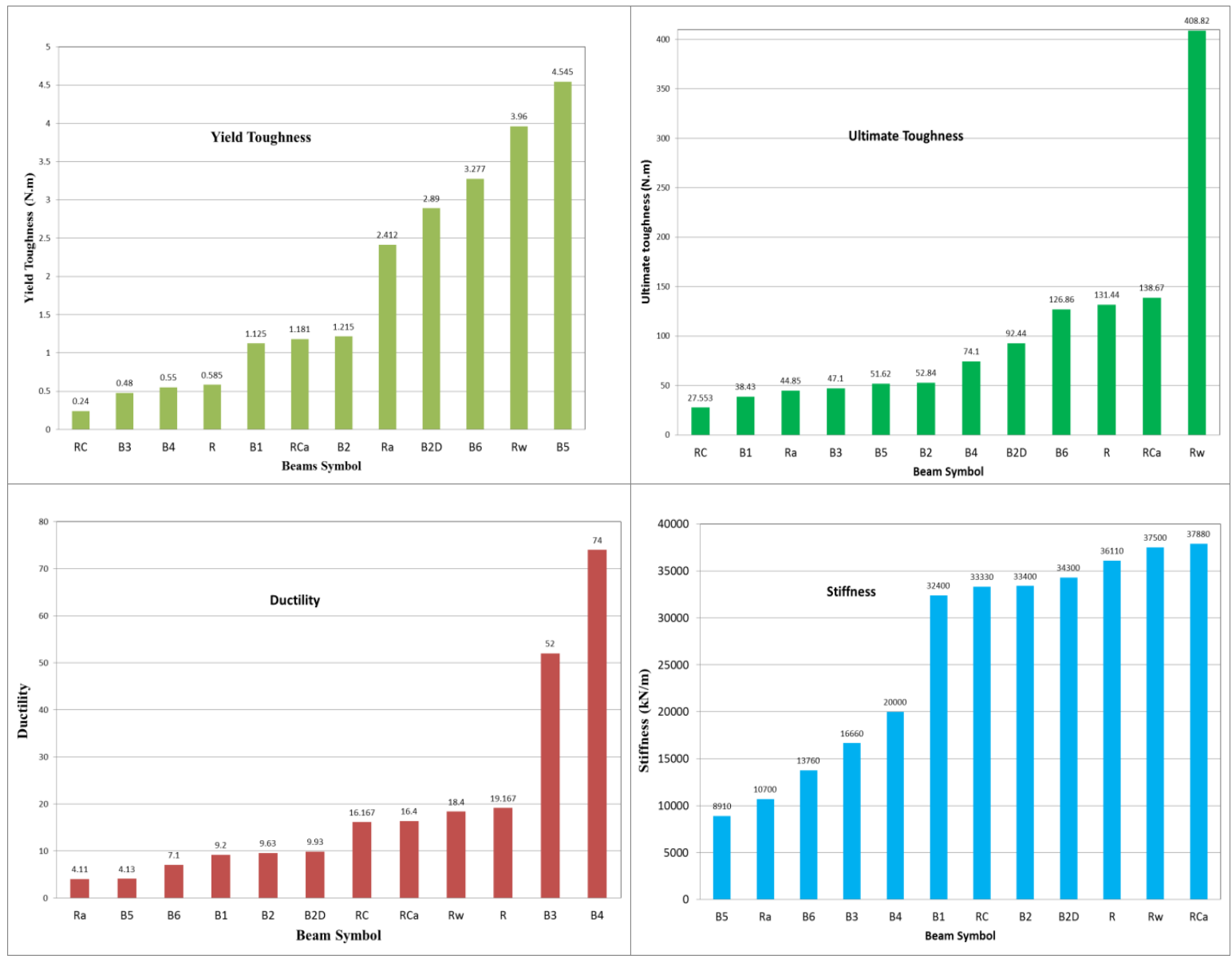

شكل (12) متانة ومطيلية وصلادة الاعتاب

المقارنة بين النتائج العملية والنظرية

وزيادة المتانة والمطيلية. اما الاعتاب الخرسانية المجوفة

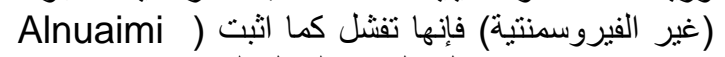
بحمل اقل من الحمل التصميمي. (et al., 2008 ب- كلما زادت عدد طبقات المشبك السلكي كلما زاد الفرق بين حمل الفنشل التصميمي و العملي. ج- الأعتاب المجوفة التي لا تحتوي على مشبك سلكي تعطي عمليا نتائج قريبة الىى النتائج النظرية.
السمنت على عكس القناني البلاستيكية والمعننية والتي

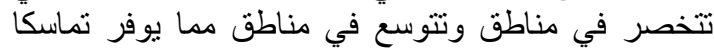

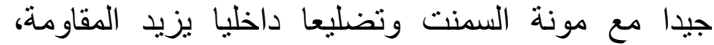
والثالث الفرق بين مرونة الانبوب البلاستيكي ومرونة

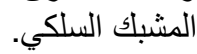


جدول (2) المقارنة بين النتائج النظرية و العملية

\begin{tabular}{|c|c|c|c|c|c|}
\hline \multicolumn{2}{|c|}{ Beam Sym. } & Type & $\mathrm{Pu}_{\mathrm{e}}(\mathrm{kN})$ & $\mathrm{Pu}_{\text {the }}(\mathrm{kN})$ & $\mathrm{Pu} / \mathrm{P}$ the \\
\hline \multirow{5}{*}{ Group 1} & $\mathrm{RC}$ & \multirow{2}{*}{$\begin{array}{c}\text { Ref. Concrete } \\
\text { beams }\end{array}$} & 22.5 & 20.974 & 1.073 \\
\hline & $\mathrm{RCa}$ & & 49.7 & 40.00 & 1.242 \\
\hline & $\mathrm{R}$ & \multirow{3}{*}{ Ref. Mortar beams } & 29 & 21.135 & 1.372 \\
\hline & $\mathrm{Ra}$ & & 32.5 & 30.65 & 1.06 \\
\hline & $R w$ & & 60.8 & --------_* & ---------- \\
\hline \multirow{3}{*}{ Group 2} & B1 & \multirow{3}{*}{ Plastic Bottle } & 25.7 & 21.135 & 1.216 \\
\hline & B2 & & 32 & 22.31 & 1.434 \\
\hline & B2D & & 35.75 & $23.16^{\star *}$ & 1.543 \\
\hline \multirow{2}{*}{ Group3 } & B3 & \multirow{2}{*}{ Metallic can } & 5.9 & 4.53 & 1.302 \\
\hline & B4 & & 7.1 & 5.86 & 1.211 \\
\hline \multirow{2}{*}{ Group 4} & B5 & \multirow{2}{*}{ Plastic Pipe } & 19.7 & 21.135 & 0.932 \\
\hline & B6 & & 39.8 & 24.59 & 1.618 \\
\hline
\end{tabular}

الطريقة المقترحة في هذا البحث تمثل تدوير عدد كبير من المن المير

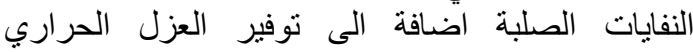
و الصوتي و الحصول على اعتاب خفيفة الوزن.

الاستتناجات

1- يمكن إنتاج أعتاب فيروسمنتية مجوف باستخدام القناني

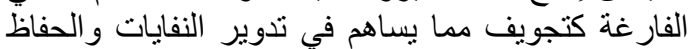

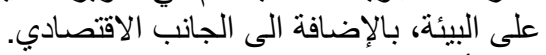

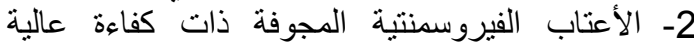

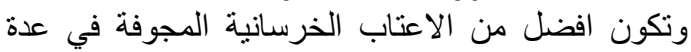
خصائص، وهي خفة الوزن و والمنانة و المطيلية العاليتين الئني

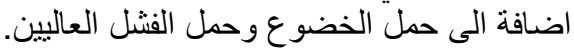

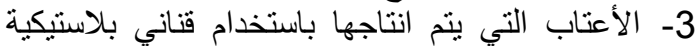

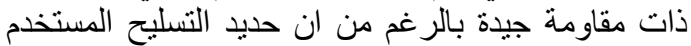

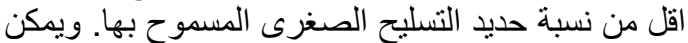

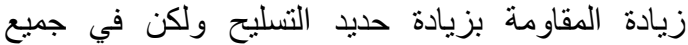
الاحو ال هنالك مقاومة بزيالية عالية باستخدام نسب القل او تساوي

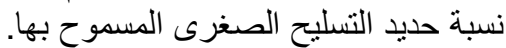
4- الأعتاب المنتجة باستخدام قناني فار غة ومشبك سلكي

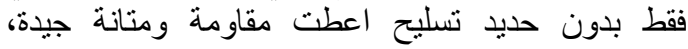
ومطيلية عالية جدا.

5- إنتاج أعتاب فيروسمنتية مجوفة يمثل طريقة كفوءة

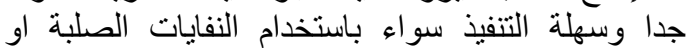

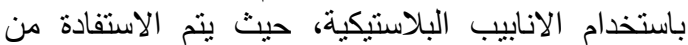

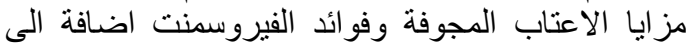

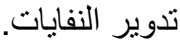

\section{التوصيات للبحوث المستقبلية} 1- دراسة نأثير قوى اللي على الاعتاب الفيروسمنتية

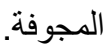

الأبنية الخضر اءء وتلوير النفايات

يشير مصطلح المباني الخضراء إلى إلى ممارسات

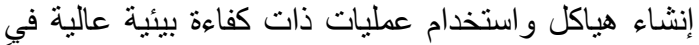
استخدام الموارد اثناء البناء وطيلة دورة حياة المنشأ، بداء

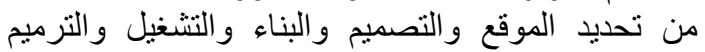

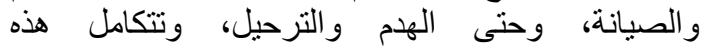

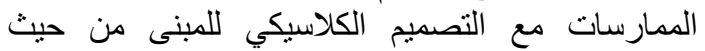

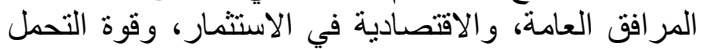

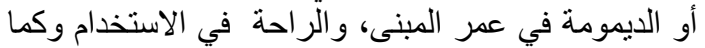

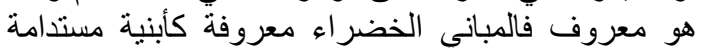

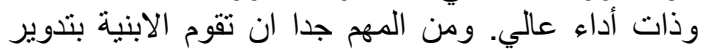

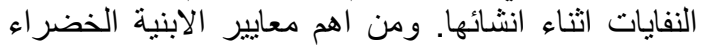
العزل الحر اري كونه يوفر طاقة التدفئة و التبريد واستخدام

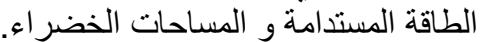

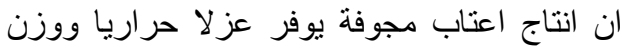

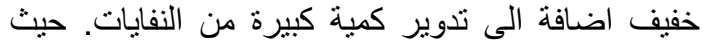
يقوم كل عتب مجوف باستخدام 3 قناني بلاستيكية كبيرة،

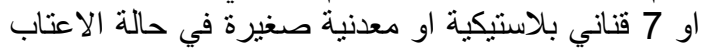

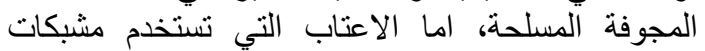

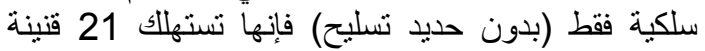
بلاستيكية او معدنبة صغيرة. ان عدد القناني البلاستيكية

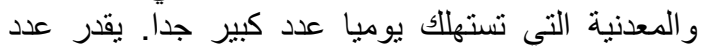
القناني المعدنية فقط (قناني المشروبات الغازية لئة) التي

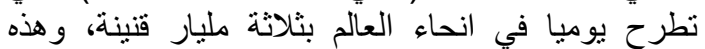

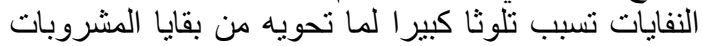

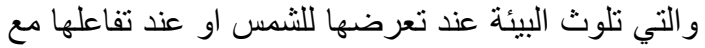

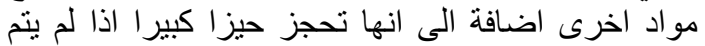
طررها بشكل دوري ومنتظم وتسبب تلوثا اذاذا لم يتم

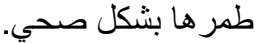


Structural Material", $\mathrm{ACl}$ Special Publication, Vol. 61, PP 9-24, Aug. 1979.

3- Al-Kubaisy, M. A., Mohd Zamin Jumaat," Strengthening of Reinforced Concrete Beams Using Ferro Cement Laminate", Concrete International, Vol. 22, Issue 10 PP 37-43, Oct. 1, 2000.

4- Falah M. Wegian and Falah A. Almottiri, "Experimental Studies on Reinforced Hollow-Block Concrete Sections", Jordan Journal of Civil Engineering, Volume 1, No. 4, 2007.

5- Ali Said Alnuaimi, Khalifa S. Al-Jabri, Abdelwahid Hago, "Comparison Between Solid and Hollow Reinforced Concrete Beams", Materials and Structures (2008) 41:269-286 DOI 10.1617/s11527-0079237-x.

6- Vesel Yazici, "Strengthening Hollow Reinforced Concrete Columns With Fiber Reinforced Polymers", Ph.D. thesis, University of Wollongong, Australia, 2012.

7- ACl-544-1R-96, "State-of-the-Art Report on Fiber Reinforced Concrete", 2010.

8- ASTM C 1018, "Standard Test Method for Flexural Toughness and First-Crack Strength of Fiber-Reinforced Concrete", 2010.

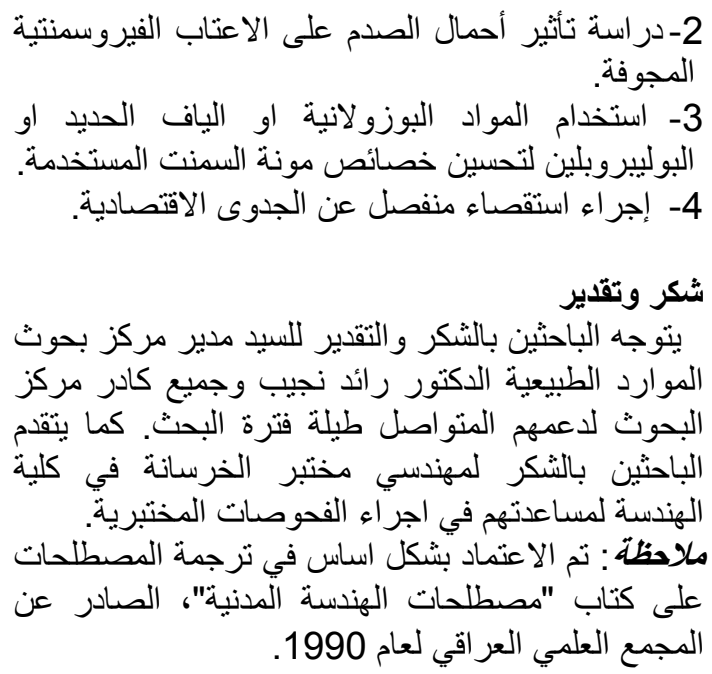

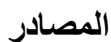

1- Naaman, Antoine E., "Design Prediction of Crack Width in Ferrocement", Ferrocement Materials and Applications, SP-61, American Concrete Institute, Detroit, 1979.

2- Gordon, B. Batson, Gajanan, M. Sabnis, and Antoine E. Naaman, "Survey of Mechanical Properties of Ferrocement as a

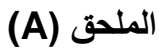
الجدول (A-1) خصائص الركام الناعم

\begin{tabular}{|c|c|c|c|c|c|}
\hline \multicolumn{4}{|c|}{ Grading of fine aggregate } & \multicolumn{3}{c|}{ Chemical and physical properties } \\
\hline $\begin{array}{c}\text { Sieve } \\
\text { size }\end{array}$ & $\begin{array}{c}\text { Cumulative } \\
\text { passing \% }\end{array}$ & $\begin{array}{c}\text { ASTM } \\
\text { C33-01 }\end{array}$ & Properties & Specification & $\begin{array}{c}\text { Test } \\
\text { Results }\end{array}$ \\
\hline $9.5-\mathrm{mm}$ & 100 & $0-10$ & Absorption \% & ASTM C128-01 & 2.3 \\
\hline $4.75-\mathrm{mm}$ & 93.25 & $95-100$ & Dry loose unit weight, kg/m3 & $\begin{array}{c}\text { ASTM } \\
\text { C29/C29M/97 }\end{array}$ & 1601 \\
\hline $2.36-\mathrm{mm}$ & 94.7 & $80-100$ & Specific gravity & ASTM C128-01 & 2.63 \\
\hline $1.18-\mathrm{mm}$ & 76.62 & $50-85$ & Sulfate content (as SO3), \% & (I.O.S.) No. 45-84 & 0.12 \\
\hline $600-\mu \mathrm{m}$ & 32.2 & $25-60$ & Material finer than 0.075 mm\% & (I.O.S.) No. 45-84 & 1.26 \\
\hline $300-\mu \mathrm{m})$ & 3.53 & $5-30$ & & & \\
\hline $150-\mu \mathrm{m}$ & 3 & $0-10$ & & & \\
\hline
\end{tabular}


الجدول (A-2) الخصائص الكيميائية والفيزيائية للركام الخشن

\begin{tabular}{|c|c|c|c|c|c|}
\hline \multicolumn{2}{|c|}{ Chemical and physical properties } & \multicolumn{3}{c|}{ Grading } \\
\hline Properties & Specification & $\begin{array}{c}\text { Test } \\
\text { Results }\end{array}$ & Sieve size & $\begin{array}{c}\text { Cumulative } \\
\text { passing \% }\end{array}$ & ASTM limit \\
\hline Specific gravity & ASTM C128-01 & 2.67 & $25.0 \mathrm{~mm}$ & 100 & 95 to 100 \\
\hline Absorption \% & ASTM C128-01 & $0.6 \%$ & $12.5 \mathrm{~mm}$ & 55 & 25 to 60 \\
\hline $\begin{array}{c}\text { Dry loose unit } \\
\text { weight, kg/m3 }\end{array}$ & $\begin{array}{c}\text { ASTM } \\
\text { C29/C29M/97 }\end{array}$ & 1590 & $4.75 \mathrm{~mm}$ & 8 & 0 to 10 \\
\hline $\mathrm{SO}_{3} \%$ & (I.Q.S.) No. 5-1984 & $0.06 \%$ & $2.36 \mathrm{~mm}$ & 3 & 0 to 5 \\
\hline
\end{tabular}

الجدول (A-3) الخصائص الكيميائية و الفيزيائية للإسمنت

\begin{tabular}{|c|c|c|c|}
\hline Description & $\begin{array}{c}\text { Content \% (Limit of } \\
\text { Iraqi specification No. } \\
5 / 1984)\end{array}$ & Description & $\begin{array}{c}\text { Content \% } \\
\text { (Limit of Iraqi } \\
\text { specification } \\
\text { No. } 5 / 1984)\end{array}$ \\
\hline Loss on Ignition & $1.73(\leq 4 \%)$ & Alumina, $\mathrm{Al}_{2} \mathrm{O}_{3}$ & 4.73 \\
\hline Silica, $\mathrm{SiO}_{2}$ & 12.78 & Insoluble material & $1.27(\leq 1.5 \%)$ \\
\hline Sulfate, $\mathrm{SO}_{3}$ & $2.13(\leq 2.8 \%)$ & $\begin{array}{c}\text { Lime Saturation Factor, } \\
(\text { L.S.F) }\end{array}$ & $0.73(0.66-1.02)$ \\
\hline Iron oxide, $\mathrm{Fe}_{2} \mathrm{O}_{3}$ & 3.15 & $\begin{array}{c}\text { Specific surface area } \\
\left(\mathrm{m}^{2} / \mathrm{kg}\right)\end{array}$ & $\begin{array}{c}367.3(>250 \\
\left.\mathrm{m}^{2} / \mathrm{kg}\right)\end{array}$ \\
\hline Magnesia, $\mathrm{MgO}$ & $3.17(\leq 5 \%)$ & Initial setting, hrs : min & $0: 48(>45 \mathrm{~min})$ \\
\hline Calcium oxide, & 65.7 & Final setting, hrs : min & $9: 00(<10 \mathrm{hrs})$ \\
\hline 3-daO f'c $\mathrm{MPa}$ & $29.3(>15 \mathrm{MPa})$ & & \\
\hline
\end{tabular}

